\title{
Fractionally quantized Berry phase, adiabatic continuation, and edge states
}

\author{
Toshikaze Kariyado* and Yasuhiro Hatsugai ${ }^{\dagger}$ \\ Division of Physics, Faculty of Pure and Applied Sciences, University of Tsukuba, Tsukuba, Ibaraki 305-8571, Japan
}

(Received 17 April 2014; revised manuscript received 1 August 2014; published 22 August 2014)

\begin{abstract}
Symmetry protected quantization of the Berry phase is discussed in relation to edge states. Assuming the existence of an adiabatic process that protects the quantization of the Berry phase, a nontrivial Berry phase $\gamma \propto 2 \pi \rho$ ( $\rho$ is the local filling of particles) for the bulk suggests appearance of edge states with boundaries. We have applied this generic consideration for the Bloch states of a two-dimensional model with massless Dirac fermions where $\gamma= \pm \pi / 2$ implies the edge states. The entanglement entropy is evaluated for the models and its relation to the bulk-edge correspondence of Dirac fermions is discussed as well.
\end{abstract}

DOI: 10.1103/PhysRevB.90.085132

PACS number(s): 73.20.-r, 03.65.Vf, 03.65.Ud

\section{INTRODUCTION}

The characterization of phases is one of the main targets of condensed matter physics. As for the description of physical states, the role of boundary conditions has been assumed to be of secondary importance. This is true when one considers classical order using an order parameter in the thermodynamic limit, since the dimensions of boundaries are less than the bulk dimension. In topological phases characterized by absence of local order parameters [1], the situation is different. In contrast to the symmetry broken phases with low-energy excitations as the Nambu-Goldstone boson, the ground state of the topological phase is mostly gapped as a bulk. With boundaries or impurities, however, low-energy excitations exist only near such a geometrical disturbance [2-8]. These edge (boundary) states characterize topological phases such as the quantum Hall systems and $Z_{2}$ topological insulators $[9,10]$. The relation between edge states and bulk topological properties is known as the bulk-edge correspondence [11,12].

In order to analyze the topological origin of edge states, an adiabatic continuation is useful. Assuming a modification of the gapped ground state of the bulk to a simple state without gap closing, one may reduce the topological properties of the physical system to those of the simple one. If that reduced system has a clear reason to have edge states, it establishes the bulk-edge correspondence. For instance, when the reduced system is composed of independent clusters and the given boundary breaks a cluster at the edge, there will be edge states interpreted as dangling states from a broken cluster at the edge $[7,13]$. On the other hand, if the boundary does not break a cluster, i.e., the boundary is in between two adjoined clusters, there is no obvious reason to have edge states. The adiabatic continuation is more powerful when it is combined with topological quantities defined by the Berry connection [14-17] using gauge twists as parameters. The Berry phase (and its generalizations) is not quantized by definition, but it can be quantized and becomes a topological quantity with the help of some symmetries [13,18,19]. When such symmetries are present, the Berry phase based argument (which will be explained later in detail) is robust against

\footnotetext{
*kariyado@rhodia.ph.tsukuba.ac.jp

†hatsugai@rhodia.ph.tsukuba.ac.jp
}

adiabatic continuation, as far as the symmetry is kept during the adiabatic continuation.

In this paper, we first give general arguments for the topological characterization of a gapped and short-range entangled state using an adiabatic continuation and the Berry phase (Sec. II). A natural interpretation of the bulk-edge correspondence in this general framework is also given. We further discuss the bulk-edge correspondence in terms of the entanglement entropy [20-24], as a complement to the Berry phase based arguments. Then, in order to demonstrate the general idea, we introduce a specific four-band model having Dirac cones in its bulk energy dispersion in Sec. III. There, we find an unusual type of the quantization of the Berry phase, a quantization into $\pm \pi / 2$, or fractional quantization, in addition to the usual quantization into $0 / \pi$. It is shown that such an unusual $\pm \pi / 2$ quantization brings a difference in the bulk-edge correspondence from the case of the usual $0 / \pi$ quantization.

\section{ADIABATIC CONTINUATION AND BULK-EDGE CORRESPONDENCE}

\section{A. Berry phase and adiabatic continuation}

Let us start our discussion from a generic lattice model of spinless fermions by a Hamiltonian with an adiabatic parameter $\lambda$ [extension with spins or for systems with $U(1)$ gauge invariance is straightforward],

$$
\begin{aligned}
H(\lambda) & =H_{E, E}+H_{L, L}+\lambda H_{L, E}, \\
H_{\alpha \beta} & =\sum_{i \in \alpha, j \in \beta}\left(c_{i}^{\dagger} t_{i j} c_{j}+\text { H.c. }+V_{i j} n_{i} n_{j}\right), \quad \alpha, \beta=L, E,
\end{aligned}
$$

where $n_{i}=c_{i}^{\dagger} c_{i}$ and the system is divided into two parts $L$ and $E$. The parameter $\lambda$ is the coupling between them (Fig. 1). It is invariant for a $U(1)$ gauge transformation $H \rightarrow H^{\prime}=$ $H$, where $c_{i} \rightarrow c_{i}^{\prime}=\Omega_{i} c_{i}$ and $t_{i j} \rightarrow t_{i j}^{\prime}=\Omega_{i} t_{i j} \Omega_{j}^{-1},\left(\left|\Omega_{i}\right|=\right.$ $1)$. We assume the many-body ground state $|G(\lambda)\rangle$ of $H(\lambda)$ is always gapped for $0 \leqslant \lambda \leqslant 1$. The physical ground state $|G(1)\rangle$ is adiabatically connected to the decoupled ground state $|G(0)\rangle$ written as

$$
|G(0)\rangle=\sum_{i_{1} i_{2} \cdots \in L} \psi_{i_{1} i_{2} \ldots}^{L} \ldots c_{i_{1}}^{\dagger} c_{i_{2}}^{\dagger} \cdots \sum_{j_{1} j_{2} \cdots \in E} \psi_{j_{1} j_{2} \cdots}^{E} c_{j_{1}}^{\dagger} c_{j_{2}}^{\dagger} \cdots|0\rangle .
$$




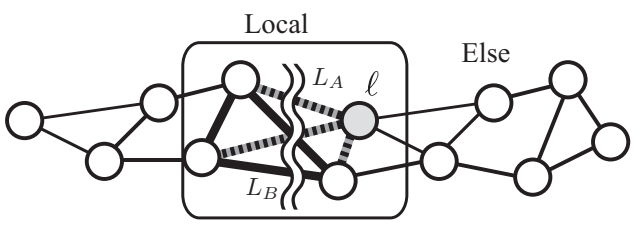

FIG. 1. General idea of the Berry phases and edge states. The hopping of the thick dotted lines is modified. When the local object $L$ is decoupled (only the hopping on the thick lines is nonzero), these gauge twists are gauged away.

It implies that the ground state $|G(1)\rangle$ is short-range entangled, that is, the ground state is composed of local quantum objects. Typical such examples are the Haldane phase of the spin 1 chain and the valence bond solid states [17,25,26]. To characterize this short-range entanglement, let us define a Berry phase $\gamma$ by introducing a gauge twist $\omega_{\ell}=e^{\mathrm{i} \theta}$, $(0 \leqslant \theta \leqslant 2 \pi)$ at some sites $\ell$ 's inside the local object $L$. ( $\ell$ 's can be multiple sites, for instance, $\ell$ s stand for the sites in $L_{A}$ in Fig. 1.) This twist dependence is given by the local Hamiltonian $H_{L, L}\left(\omega_{\ell}\right)$ where the hopping $t_{i \ell}$ connecting the sites $\ell$ 's and the remained sites $i$ 's inside the local object $L$ are replaced by $t_{i \ell} \omega_{\ell}$. Note that this gauge twist does not affect any coupling in $H_{L, E}$. Then the Berry phase defined below characterizes the locality of the gapped phase

$$
\mathrm{i} \gamma=\int\langle G \mid d G\rangle=\int_{0}^{2 \pi} d \theta\left\langle G_{\theta} \mid \partial_{\theta} G_{\theta}\right\rangle \bmod 2 \pi .
$$

As for the decoupled case $(\lambda=0)$, this $\gamma$ is evaluated as

$$
\gamma=-2 \pi \bar{\rho} \bmod 2 \pi \text {, }
$$

where

$$
\bar{\rho}=\sum_{\ell}\left\langle G_{\theta=0}(0)\left|n_{\ell}\right| G_{\theta=0}(0)\right\rangle
$$

is the sum of an averaged filling of the fermion at the site $\ell$ since the gauge twist $\omega_{\ell}$ is gauged out by the gauge transformation [27] (see Appendix A). If there exists some symmetry (such as the chiral symmetry, reflection, time reversal) to guarantee the quantization of the Berry phase $\gamma$, this Berry phase is an adiabatic invariant and it is used for a topological order parameter at the physical point $\lambda=1$. We have many successful examples for such situations [17,28-30]. Even if such a symmetry is absent, we may still expect that a substantially large value of $\gamma$ implies the existence of the short-range entanglement. That is, the Berry phase $\gamma$ can be a good topological order parameter.

For a gapped phase that can be well described by a collection of local objects $L$, a finite $\gamma$ suggests appearance of edge states when the boundary is on the gauge twisted bonds. In the decoupled limit, such a boundary breaks a local object and broken pieces appear as edge states [31]. Even for a finite coupling, the edge states can be still localized with the symmetry protection, since the Berry phase $\gamma$ is an adiabatic invariant and the locality of the ground state retains as well. This general idea can be applied to several systems. Application is not limited to one-dimensional systems and it is applicable to higher dimensions. When the system is free of many-body interactions, the momenta parallel to a given edge (or surface) are regarded as parameters determining an effective one-dimensional model. One of such important examples is a zero-mode edge state at the zigzag boundary of graphene, which is characterized by the Berry phase in the effective one-dimensional model $[13,32,33]$. The quantization of the Berry phase $\gamma / \pi \in \mathbb{Z}$ is well known today but here in this paper, we demonstrate $\gamma= \pm \pi / 2$, i.e., a fractional quantization of $\gamma$ is also useful for the bulk-edge correspondence of the Dirac fermions.

Our arguments so far are developed to interpret the connection between bulk topological characters and edge states assuming that a proper adiabatic continuation to the simple model is known. To predict the existence of edge states for a given boundary without the knowledge of adiabatic continuation, we should apply a gauge twist on the bonds that cross that given boundary. Then, as far as there exist an adiabatic continuation to the model with decoupled local objects (we do not have to know the explicit way to achieve it), the Berry phase criterion for the existence of edge states is still valid.

\section{B. Entanglement entropy and adiabatic continuation}

The locality of the gapped ground state is also reflected in the entanglement entropy for spatial bipartition by a given boundary. Namely, the entanglement entropy can detect how the local object is broken by a given boundary as the Berry phase. Since the entanglement entropy is a bulk quantity, it provides another view of the bulk-edge correspondence. Especially, the behavior of the entanglement entropy against the adiabatic continuation to the $\lambda=0$ limit is important [23]. If the entanglement entropy is continuously reduced to 0 as $\lambda$ goes to 0 , it implies there is no obvious reason to have edge states, while if it remains finite in the $\lambda=0$ limit, there are dangling states that are observed as edge states. Then, the entanglement entropy and the Berry phase are directly related through edge states [20].

For the noninteracting case with one fermion in $L$, we can demonstrate this explicitly. In the $\lambda=0$ limit, there is no need to consider the entanglement between $L$ and $E$. Then, the entanglement entropy for the bipartition of $L$ into two parts, $L_{A}$ and $L_{B}$, is obtained as

$$
S=-[\bar{\rho} \ln \bar{\rho}+(1-\bar{\rho}) \ln (1-\bar{\rho})],
$$

where $\bar{\rho}$ is the fermion filling in $L_{A}$ (see Appendix B). Just as in the case of the Berry phase, it is determined from $\bar{\rho}$ only. $S$ in Eq. (5) takes maximum when $\bar{\rho}=0.5$, which corresponds to $\gamma=\pi$.

\section{EXAMPLE IN A FERMIONIC MODEL}

\section{A. Model and methods}

As an example to describe the general ideas explained above, we introduce a spinless fermionic tight-binding model having four sublattices in a unit cell [see Fig. 2(a)]. We define three kinds of transfer integrals, $t_{0}, t_{1}$, and $t_{2}$ as in Fig. 2(a), and set $t_{0}=1.0, t_{1}=0.5$, and $t_{2}=1.5$ throughout this paper. The properties of a very similar model have been addressed in Ref. [34] very recently, and thus, we concentrate on the Berry phase and its fractional quantization in this paper. For the edge 

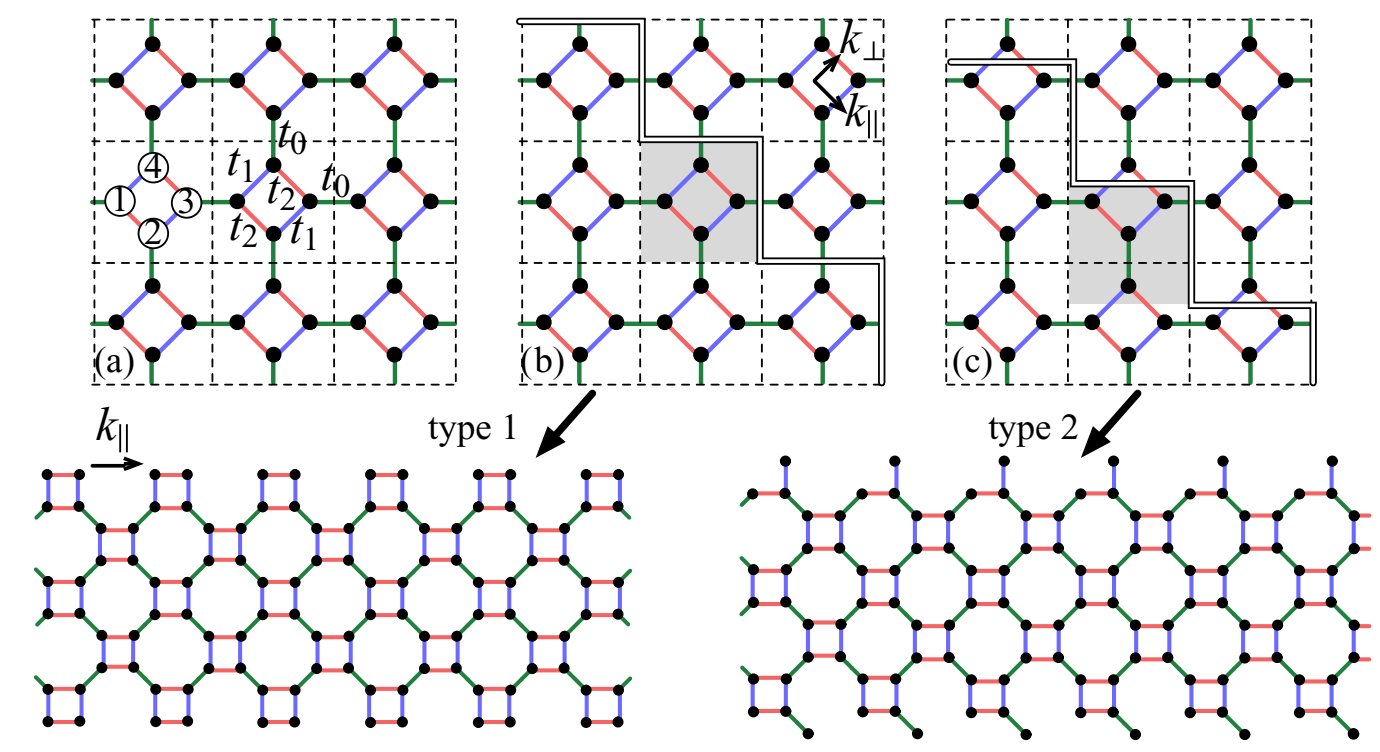

FIG. 2. (Color online) (a) Definitions of transfer integrals. Labels of sublattices are also shown. (b) and (c) Definitions of type 1 and type 2 edges. Shaded regions are the corresponding unit cells. Examples of ribbons for edge spectrum calculations are shown in the lower panels.

state characterization, we consider two kinds of edge shapes named as type 1 and type 2 [see Figs. 2(b) and 2(c)]. In order to treat a two-dimensional model, we introduce momenta parallel $\left(k_{\|}\right)$and perpendicular $\left(k_{\perp}\right)$ to the edge whose directions are shown in Fig. 2(b), with $k_{\|}$acting as a parameter determining an effective one-dimensional model. In the present case, the gauge twist $\omega_{\ell}=\mathrm{e}^{\mathrm{i} \theta}$ can be regarded as a twisted boundary condition, and the integration over $\theta$ is essentially equivalent to the integration over $k_{\perp}$. Then, the Berry phase (2) is given by the Zak phase [35]

$$
\mathrm{i} \gamma\left(k_{\|}\right)=\sum_{n \in \text { filled }} \int_{-\pi}^{\pi} d k_{\perp}\left\langle u_{n k_{\perp} k_{\|}}\left|\partial_{k_{\perp}}\right| u_{n k_{\perp} k_{\|}}\right\rangle,
$$

and we use this expression for computational convenience. Here, $k_{\perp}$ is properly scaled so that the Bloch wave function $\left|u_{n k_{\perp} k_{\|}}\right\rangle$has periodicity $2 \pi$ in $k_{\perp}$. Note that a unit cell convention is directly related to $\gamma\left(k_{\|}\right)$. For each boundary shape, we set a unit cell so that the given boundary lies in between two neighboring unit cells [19] to use the Berry phase $\gamma\left(k_{\|}\right)$to discuss the edge states. [See shaded regions in Figs. 2(b) and 2(c).] We employ the technique in Refs. [36] and [27] for the Berry phase calculations. The edge spectrum is calculated with a ribbon geometry like those in the lower panels of Fig. 2, though the actual calculations are performed on much wider ribbons. In order to discuss the fractional quantization, we focus on the quarter filling case, the case of one fermion per unit cell, throughout this paper.

\section{B. Fractional quantization of the Berry phase and the bulk-edge correspondence}

Figure 3 shows the edge spectra (upper panels) and the Berry phases (lower panels) for the type-1 (a) and type-2 (b) edges. As we handle the quarter filling case, we should focus on the lowest band and the gap just above it. For the given parameter set, Dirac cones appear between the lowest and second lowest bands in the bulk energy dispersion.
Consequently, the bulk continuum, the region filled with bands with bulk nature, touches at two points, which are projected Dirac cones. For the type- 1 edge, the Berry phase is quantized into 0 and $\pi$. On the other hand, for the type- 2 edge, the Berry phase is quantized into $\pm \pi / 2$, i.e., fractional quantization is really achieved. As we have noted, the Berry phase is related to the site-resolved filling $\bar{\rho}$. [Eq. (3) for $\lambda=0$.] Here, for the two-dimensional case, the sublattice and $k_{\|}$-resolved filling $\rho_{a}\left(k_{\|}\right)$play the role of $\bar{\rho}$. For the present model, the symmetries impose restrictions and force $\rho_{a}\left(k_{\|}\right)$to take a fixed value. This is essential for observing the fractional quantization. More specifically, the time reversal symmetry gives

$$
\rho_{a}\left(-k_{\|}\right)=\rho_{a}\left(k_{\|}\right),
$$

the inversion symmetry gives

$$
\rho_{1}\left(-k_{\|}\right)=\rho_{3}\left(k_{\|}\right), \quad \rho_{2}\left(-k_{\|}\right)=\rho_{4}\left(k_{\|}\right),
$$

and the mirror symmetry gives

$$
\rho_{1}\left(-k_{\|}\right)=\rho_{2}\left(k_{\|}\right), \quad \rho_{3}\left(-k_{\|}\right)=\rho_{4}\left(k_{\|}\right) .
$$
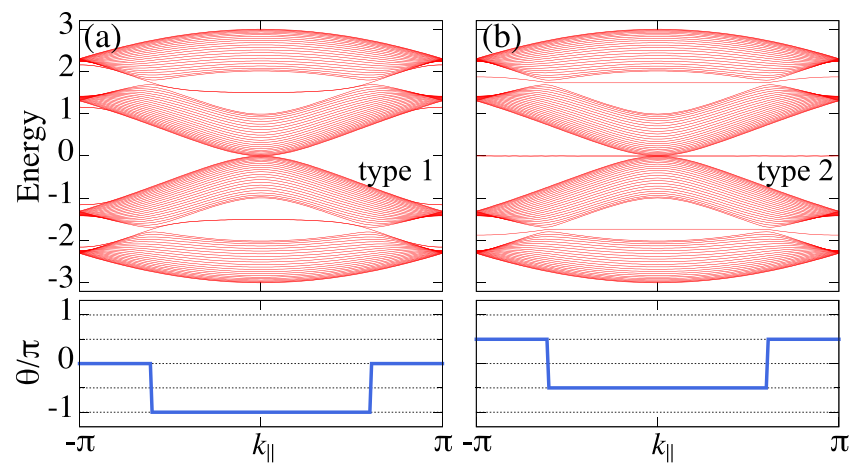

FIG. 3. (Color online) Energy spectra and Berry phases for quarter filling for type-1 (a) and type-2 (b) edges. 
In addition, $\sum_{a} \rho_{a}\left(k_{\|}\right)=1$ for the quarter filling. Combining these relations, we finally obtain the relation $\rho_{a}\left(k_{\|}\right)=1 / 4$, which leads to the fractional quantization of the Berry phase into $\pm \pi / 2$. As this derivation shows, the $\pm \pi / 2$ quantization is a kind of symmetry protection by crystal symmetries. Here, we have demonstrated the fractional quantization in a specific model. However, the idea is generally applicable to any model in which the sublattice-resolved particle filling $\bar{\rho}$ [or, $\rho_{a}\left(k_{\|}\right)$] is fixed by some symmetry.

The fractional quantization into $\pm \pi / 2$ brings a difference in the bulk-edge correspondence from the case of $0 / \pi$ quantization. For the type- 1 edge where $0 / \pi$ quantization is observed, an edge state distinct from the bulk continuum exists as they connect two projected Dirac cones. In the region with the edge states, $\gamma\left(k_{\|}\right)$takes the value of $\pi(-\pi$ is equivalent to $\pi$ ). The existence or absence of the edge state are switched at the gap closing point, which is consistent with a $\pi$ jump in $\gamma\left(k_{\|}\right)$[19]. In this case, the edge states are doubly degenerate and they are localized at the left and right boundaries, respectively. As is well known, the Berry phase is related to bulk polarization [36]. Then, the degeneracy between the left and right edge states is due to the equivalence between $\gamma=\pi$ and $\gamma=-\pi$, which implies that there is left/right symmetry and no favored direction of polarization. On the other hand, edge states appearing for the type-2 edge, for which $\pm \pi / 2$ quantization takes place, do not have such symmetry. There appears only one nondegenerate edge state through the entire edge Brillouin zone. In this case, the spatial position of the edge states is switched at the projected Dirac cone. Namely, the edge state near $k_{\|}=0$ lives on the edge at the one side of the ribbon, say the left edge, while the one near $k_{\|}=\pi$ lives on the edge at the other side. This difference in the spatial position is a consequence of the fractional Berry phase $\pm \pi / 2$, that is, $\gamma=\pi / 2$ and $\gamma=-\pi / 2$ correspond to the polarizations in opposite direction.

\section{Interpretation with adiabatic continuation}

Here, we apply adiabatic continuation focusing on the effective one-dimensional model for $k_{\|}=0$ and $\pi$. Schematically, this one-dimensional model is illustrated in Fig. 4(a), in which a plus (minus) sign should be taken for the transfer integral denoted as $\pm t_{0}$ for $k_{\|}=0\left(k_{\|}=\pi\right)$. The type- 1 and type- 2 edges and the corresponding unit cell conventions in the effective one-dimensional model are indicated as doubled lines and shaded regions in Fig. 4. For $k_{\|}=0$, the model can be adiabatically connected, i.e., smoothly transformed without closing the gap between the lowest and second lowest bands, to the model in Fig. 4(b) by replacing $t_{1}$ by $\lambda t_{1}$ and reducing $\lambda$ from 1 to 0 gradually. Note that the model in $\lambda \rightarrow 0$ limit is composed of decoupled clusters, or local objects, and this operation maintains the symmetry of the model and the Berry phase quantization. For $k_{\|}=\pi$, a different adiabatic continuation must be applied to keep the gap, namely, we should replace $t_{0}$ by $\lambda t_{0}$ and take $\lambda \rightarrow 0$ limit. This operation results in a model in Fig. 4(d).

Next, we explicitly show that edge states are induced by breaking local objects. For $k_{\|}=0$, the energy levels in the $\lambda \rightarrow 0$ limit are $t_{2}+t_{0}, t_{2}-t_{0},-t_{2}+t_{0}$, and $-t_{2}-t_{0}$. Then, if the type 2 edge is introduced here, it breaks the local object at
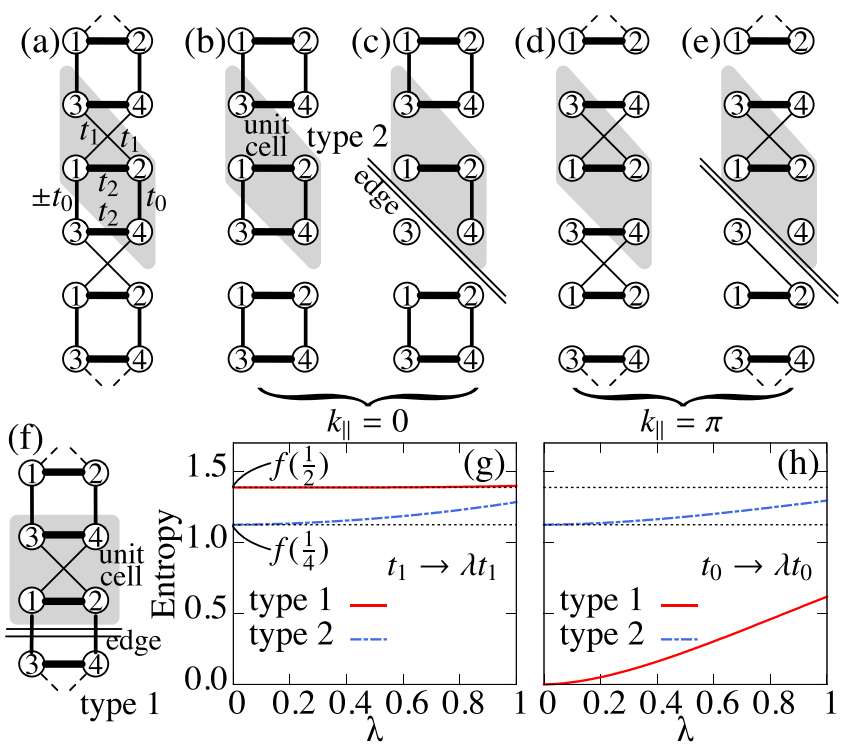

FIG. 4. (Color online) (a) One-dimensional model for a fixed $k_{\|}$. For the transfer integral indicated as $\pm t_{0}$, take $+t_{0}\left(-t_{0}\right)$ for $k_{\|}=0$ $(\pi)$. (b) and (d) Models obtained after the adiabatic continuation. (c) and (e) Models obtained after the adiabatic continuation with the edge. (b) and (c) are for $k_{\|}=0$, while (d) and (e) are for $k_{\|}=\pi$, respectively. (g) and (h) Entanglement entropy for $k_{\|}=0(\mathrm{~g})$ and $k_{\|}=\pi(\mathrm{h})$. A function $f(x)$ is defined as $f(x)=-2[x \ln x+(1-$ $x) \ln (1-x)]$.

the edge as Fig. 4(c), and modifies the energy levels to $\pm\left(t_{2}^{2}+\right.$ $\left.t_{0}^{2}\right)^{1 / 2}$ and 0 (doubly degenerate). Since $-t_{2}-t_{0}<-\left(t_{2}^{2}+\right.$ $\left.t_{0}^{2}\right)^{1 / 2}<-t_{2}+t_{0}$, the state with energy $-\left(t_{2}^{2}+t_{0}^{2}\right)^{1 / 2}$ appears as an ingap edge state in Fig. 4(b) near $k_{\|}=0$. The wave function for this ingap state has its weight only in the upper side of the doubled line in Fig. 4(c). In exactly the same way, the origin of the edge state near $k_{\|}=\pi$ in Fig. 3(b) can be identified, but due to the difference in the adapted adiabatic continuation, the wave function for the ingap state has its weight only in the lower side of the doubled line in Fig. 4(e), which is opposite from the case of $k_{\|}=0$.

Figure 4 also shows the entanglement entropy for $k_{\|}=0$ and $\pi$. In practice, the entanglement entropy is numerically calculated using the formula based on the correlation function $\left\langle c_{i}^{\dagger} c_{j}\right\rangle$ [37] (see Appendix B), and arranging the effective onedimensional model in a closed circle shape and inserting two cuts to perform bipartition. Here, two cuts are required to divide a closed circle into two parts, and two local objects (in the decoupled limit) are broken in this procedure. We consider two kinds of cutting shapes corresponding to the type- 1 and type- 2 boundaries. We plot $\lambda$ dependence of the entanglement entropy for $k_{\|}=0$ [Fig. 4(g)] and $\pi$ [Fig. 4(h)], because the way the entanglement entropy behaves under the adiabatic continuation is important for the bulk-edge correspondence as we have noted. For $k_{\|}=0$, the entanglement entropy is finite in the $\lambda \rightarrow 0$ limit for both of the type- 1 and type- 2 edges, which nicely fits the observation of the edge states for $k_{\|}=0$ for both types of edges in Fig. 3. On the other hand, for $k_{\|}=\pi$, the entanglement entropy in the $\lambda \rightarrow 0$ limit is finite only for the type- 2 edge, and zero for the type-1 edge. Again, this result 
fits the appearance (absence) of the edge state for $k_{\|}=\pi$ for the type-1 (type-2) edge in Fig. 3. Figures 4(g) and 4(h) also indicate that the entanglement entropy in the $\lambda \rightarrow 0$ limit is really derived by Eq. (5) with an extra factor of two coming from our procedure to make a bipartition in which two local objects are broken.

\section{SUMMARY}

To summarize, we develop a general theory to characterize a gapped and short-range entangled state on the basis of adiabatic continuation and the Berry phase. We give a natural interpretation of the bulk-edge correspondence with the idea of a broken local object. The relation between the Berry phase and the entanglement entropy in a specific limit is also pointed out. In the latter half, these general ideas are tested in a model with Dirac cones. We find a new type of the Berry phase quantization, the quantization into $\pm \pi / 2$ in the introduced model. It is also shown that the new type of quantization modifies the way an edge state emerges from the case of usual $0 / \pi$ quantization.

\section{ACKNOWLEDGMENTS}

This work is partly supported by Grants-in-Aid for Scientific Research Nos.26247064, 25610101, and 25107005 from JSPS.

\section{APPENDIX A: DERIVATION OF EQ. (3)}

Let us consider a case in which we divide $L$ into $L_{A}$ and $L_{B}$, and apply a gauge twist by choosing sites in $L_{A}$ as $\ell$ s (in Sec. II A). With this gauge twist, the $t_{i \ell}\left(\ell \in L_{A}\right.$, $i \in L_{B}$ ) appearing in $H_{L, L}$ is modified as $t_{i \ell} \rightarrow t_{i \ell} \omega_{\ell}=t_{i \ell} \mathrm{e}^{\mathrm{i} \theta}$. For $\lambda=0$, the transformation by this gauge twist is written as a unitary transformation [27] $H_{L, L} \rightarrow U_{\theta} H_{L, L} U_{\theta}^{\dagger}$ with $U_{\theta}=\exp \left(-\mathrm{i} \theta \sum_{i \in L_{A}} n_{i}\right.$ ), where $n_{i}=c_{i}^{\dagger} c_{i}$. (Note that if $\lambda$ is finite, $U_{\theta}$ also causes an unwilling transformation of $H_{L, E}$, since $H_{L, E}$ may contain hopping terms involving sites in $L_{A}$, which means that the following argument cannot be applied for finite $\lambda$.) This means that the ground-state wave function for finite $\theta,\left|G_{\theta}(0)\right\rangle$, can be written as $\left|G_{\theta}(0)\right\rangle=U_{\theta}\left|G_{\theta=0}(0)\right\rangle$. Substituting this wave function to Eq. (2), we have

$$
\begin{aligned}
\mathrm{i} \gamma & =\int_{0}^{2 \pi} \mathrm{d} \theta\left\langle G_{\theta=0}(0)\left|\left(U_{\theta}^{\dagger} \partial_{\theta} U_{\theta}\right)\right| G_{\theta=0}(0)\right\rangle \\
& =-2 \pi \mathrm{i}\left\langle G_{\theta=0}(0)\left|\sum_{i \in L_{A}} n_{i}\right| G_{\theta=0}(0)\right\rangle,
\end{aligned}
$$

with ambiguity of integer multiples of $2 \pi$, which proves Eq. (3) for $\lambda=0$.

\section{APPENDIX B: DERIVATION OF EQ. (5)}

Here, we consider the entanglement entropy in a noninteracting fermion system on an $N$-site lattice. Now, we divide the $N$-site system into two parts, $A$ and $B$ containing $N_{A}$ and $N_{B}$ sites, respectively. Then, the entanglement entropy for this bipartition is obtained as [37]

$$
S=-\sum_{\alpha}\left[\xi_{\alpha} \ln \xi_{\alpha}+\left(1-\xi_{\alpha}\right) \ln \left(1-\xi_{\alpha}\right)\right],
$$

where $\xi_{\alpha}$ is eigenvalues of $N_{A} \times N_{A}$ matrix $\hat{C}$, whose elements are defined as $C_{i j}=\left\langle c_{j}^{\dagger} c_{i}\right\rangle(i, j \in A)$. In practice, $C_{i j}$ is calculated as

$$
C_{i j}=\sum_{E_{\alpha}<E_{F}} \psi_{i}^{(\alpha)} \psi_{j}^{(\alpha) *} \quad(i, j \in A),
$$

where $\psi_{i}^{(\alpha)}$ is the wave function with energy $E_{\alpha}$. Here, we define a vector $\vec{\psi}_{\alpha}$ whose dimension is $N_{A}$ and the elements are $\psi_{i}^{(\alpha)}(i \in A)$. Then, $\hat{C}$ is written as

$$
\hat{C}=\sum_{E_{\alpha}<E_{F}} \vec{\psi}_{\alpha} \vec{\psi}_{\alpha}^{\dagger}
$$

Now, we apply these results to the situation in Sec. II, especially focusing on the $\lambda=0$ limit and the noninteracting case. For $\lambda=0$, there is no need to consider the entanglement between $L$ and $E$, which means that what we have to calculate is the entanglement entropy for the bipartition of $L$ into $L_{A}$ and $L_{B}$. In this case, the wave function of $H_{L, L}, \varphi_{i}^{(\alpha)}$, can be used in Eq. (B2) instead of $\psi_{i}^{(\alpha)}$, the wave function of the full Hamiltonian. Then, if we further assume that there is only one fermion in $L$, we have

$$
\hat{C}=\vec{\varphi}_{0} \vec{\varphi}_{0}^{\dagger}
$$

because there is only one state below $E_{F}$ in $L$ under the constraints of one fermion in $L$. Here, as $\vec{\psi}_{\alpha}, \vec{\varphi}_{0}$ is a vector with its components being $\varphi_{i}^{(0)}\left(i \in L_{A}\right)$, where $\varphi_{i}^{(0)}$ is the ground-state wave function for $H_{L, L}$. The eigenvalues of $\hat{C}$ in Eq. (B4) can be easily obtained, namely, $\vec{\varphi}_{0}$ is an eigenvector with eigenvalue $\left|\vec{\varphi}_{0}\right|^{2}$, and any other vectors orthogonal to $\vec{\varphi}_{0}$, which can be constructed by Schmidt orthogonalization, for instance, the eigenvector with eigenvalue 0 . As a consequence, we have

$$
S=-\left|\vec{\varphi}_{0}\right|^{2} \ln \left|\vec{\varphi}_{0}\right|^{2}-\left(1-\left|\vec{\varphi}_{0}\right|^{2}\right) \ln \left(1-\left|\vec{\varphi}_{0}\right|^{2}\right) .
$$

However, $\left|\vec{\varphi}_{0}\right|^{2}$ is nothing more than the total weight of the wave function in $L_{A}$, and this proves Eq. (5).
[1] X. G. Wen, Phys. Rev. B 40, 7387 (1989).

[2] B. I. Halperin, Phys. Rev. B 25, 2185 (1982).

[3] W. P. Su, J. R. Schrieffer, and A. J. Heeger, Phys. Rev. Lett. 42, 1698 (1979).
[4] C.-R. Hu, Phys. Rev. Lett. 72, 1526 (1994).

[5] Y. Tanaka and S. Kashiwaya, Phys. Rev. Lett. 74, 3451 (1995).

[6] M. Fujita, K. Wakabayashi, K. Nakada, and K. Kusakabe, J. Phys. Soc. Jpn. 65, 1920 (1996). 
[7] A. Y. Kitaev, Physics-Uspekhi 44, 131 (2001).

[8] G. E. Volovik, The Universe in a Helium Droplet (Clarendon Press, Oxford, 2003).

[9] M. Z. Hasan and C. L. Kane, Rev. Mod. Phys. 82, 3045 (2010).

[10] X.-L. Qi and S.-C. Zhang, Rev. Mod. Phys. 83, 1057 (2011).

[11] Y. Hatsugai, Phys. Rev. Lett. 71, 3697 (1993).

[12] Y. Hatsugai, Phys. Rev. B 48, 11851 (1993).

[13] S. Ryu and Y. Hatsugai, Phys. Rev. Lett. 89, 077002 (2002).

[14] M. V. Berry, Proc. R. Soc. A 392, 45 (1984).

[15] Y. Hatsugai, J. Phys. Soc. Jpn. 73, 2604 (2004).

[16] Y. Hatsugai, J. Phys. Soc. Jpn. 74, 1374 (2005).

[17] Y. Hatsugai, J. Phys. Soc. Jpn. 75, 123601 (2006).

[18] Y. Hatsugai, New J. Phys. 12, 065004 (2010).

[19] T. Kariyado and Y. Hatsugai, Phys. Rev. B 88, 245126 (2013).

[20] S. Ryu and Y. Hatsugai, Phys. Rev. B 73, 245115 (2006).

[21] M. Arikawa, S. Tanaya, I. Maruyama, and Y. Hatsugai, Phys. Rev. B 79, 205107 (2009).

[22] M.-C. Chung, Y.-H. Jhu, P. Chen, and S. Yip, Europhys. Lett. 95, 27003 (2011).

[23] T. L. Hughes, E. Prodan, and B. A. Bernevig, Phys. Rev. B 83, 245132 (2011).

[24] A. Chandran, M. Hermanns, N. Regnault, and B. A. Bernevig, Phys. Rev. B 84, 205136 (2011).
[25] X. Chen, Z.-C. Gu, and X.-G. Wen, Phys. Rev. B 82, 155138 (2010).

[26] F. Pollmann, E. Berg, A. M. Turner, and M. Oshikawa, Phys. Rev. B 85, 075125 (2012).

[27] T. Hirano, H. Katsura, and Y. Hatsugai, Phys. Rev. B 78, 054431 (2008).

[28] I. Maruyama and Y. Hatsugai, J. Phys. Soc. Jpn. 76, 113601 (2007).

[29] T. Hirano, H. Katsura, and Y. Hatsugai, Phys. Rev. B 77, 094431 (2008).

[30] I. Maruyama, T. Hirano, and Y. Hatsugai, Phys. Rev. B 79, 115107 (2009).

[31] When the boundary allows multiple edge states within a finite region, residual interaction among the edge states can be effective, which makes the edge states gapped.

[32] Y. Hatsugai, Solid State Commun. 149, 1061 (2009).

[33] P. Delplace, D. Ullmo, and G. Montambaux, Phys. Rev. B 84, 195452 (2011).

[34] Y. Yamashita, M. Tomura, Y. Yanagi, and K. Ueda, Phys. Rev. B 88, 195104 (2013).

[35] J. Zak, Phys. Rev. Lett. 62, 2747 (1989).

[36] R. D. King-Smith and D. Vanderbilt, Phys. Rev. B 47, 1651 (1993).

[37] I. Peschel and V. Eisler, J. Phys. A: Math. Theor. 42, 504003 (2009). 